Published in final edited form as:

Biochemistry. 2016 October 11; 55(40): 5649-5652. doi:10.1021/acs.biochem.6b00920.

\title{
The AAA+ FtsH protease degrades an ssrA-tagged model protein in the inner membrane of $E$. coli
}

\author{
Sanjay B. Hari and Robert T. Sauer ${ }^{\star}$ \\ Department of Biology, Massachusetts Institute of Technology, Cambridge, MA 02139
}

\begin{abstract}
In eubacteria, the tmRNA system frees ribosomes that stall during protein synthesis and adds an ssrA tag to the incompletely translated polypeptide to target it for degradation. The AAA+ClpXP protease degrades most ssrA-tagged proteins in the E. coli cytoplasm and was recently shown to degrade an ssrA-tagged protein in the inner membrane. However, we find that tmRNA-mediated tagging of E. coli ProW $_{1-182}$, a different inner-membrane protein, results in degradation by the membrane-tethered AAA+ FtsH protease. ClpXP played no role in degradation of $\mathrm{ProW}_{1-182}$ in vivo. These studies suggest that a complex distribution of proteolytic labor maintains protein quality control in the inner membrane.
\end{abstract}

\section{Graphical Abstract}

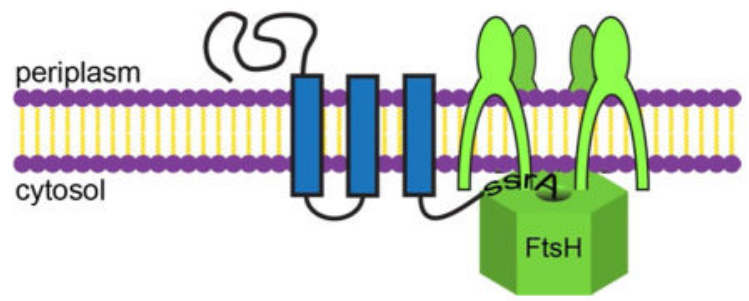

Ribosomes that stall during protein synthesis are a common source of defective intracellular polypeptides. ${ }^{1}$ In addition to producing an incomplete polypeptide, stalling depletes the pool of ribosomes available for translation and inhibits normal growth. In Escherichia coli and most eubacteria, the hybrid transfer-messenger RNA (tmRNA) system rescues stalled ribosomes and simultaneously appends a short sequence of C-terminal amino acids, called the ssrA tag, that targets the incomplete protein for degradation by ATP-fueled AAA+ proteases in the cytoplasm and by energy-independent proteases in the periplasm. ${ }^{2,3}$ For example, $\sim 10 \%$ of $E$. coli translation events appear to terminate with tmR-NA rescue and ssrA tagging, ${ }^{1,4}$ but our understanding of how these ssrA-tagged aberrant proteins are

*Corresponding Author: bobsauer@mit.edu.

Author Contributions

S.B.H. designed and performed all experiments. S.B.H. and R.T.S. contributed to data analysis and writing the manuscript.

The authors declare no competing financial interests.

Supporting Information The Supporting Information is available free of charge on the ACS Publications website. 
degraded in different cellular compartments is incomplete, especially for membrane proteins.

E. coli contains five AAA+ proteases: ClpXP, ClpAP, FtsH, HslUV, and Lon, each consisting of a multimeric AAA+ ring that binds, unfolds, and translocates protein substrates into a degradation chamber formed by attached domains or separate peptidase subunits. ${ }^{5}$ Cytoplasmic ssrA-tagged proteins are principally degraded by the ClpXP protease, with some help from ClpAP. ${ }^{2,6,7} \mathrm{FtsH}$, which is anchored to the cytoplasmic face of the inner membrane, degrades soluble ssrA-tagged proteins in vitro and has been shown to degrade several integral membrane proteins in vivo. ${ }^{8-11}$ Thus, it has been widely assumed that FtsH is also responsible for degrading ssrA-tagged membrane proteins. ${ }^{12}$ Challenging this assumption, however, a recent study showed that ClpXP degrades ssrA-tagged AcrB, an $E$. coli inner-membrane protein. ${ }^{13}$ Here, we investigate a different ssrA-tagged integral membrane protein and find that its degradation requires FtsH but not ClpXP or ClpAP in vivo. Thus, which AAA+ protease degrades which ssrA-tagged inner-membrane protein depends on factors that are currently poorly defined.

E. coli ProW is a multiple-pass inner-membrane protein. ${ }^{14}$ For our studies, we used a truncated variant $\left(\mathrm{ProW}_{1-182}\right)$ with an $\mathrm{N}$-terminal periplasmic domain, three transmembrane segments, and a C-terminus (with two added lysines) predicted to be cytosolic. ${ }^{15} \mathrm{ProW}_{1-182}$

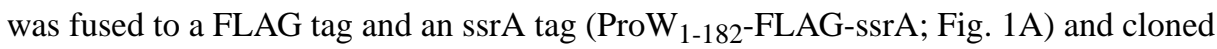
under control of an IPTG-inducible $P_{\text {trc }}$ promoter. We also generated an otherwise identical construct in which the last two residues of the ssrA tag were replaced with aspartic acids (ssrA(DD)), as this mutant tag is not recognized by cellular proteases. ${ }^{3}$ In ${ }^{35} \mathrm{~S}$ pulse-chase experiments (Table 1 and Fig. 1B), ProW ${ }_{1-182}$-FLAG-ssrA was degraded quickly $\left(\mathrm{t}_{1 / 2} \sim 5\right.$ $\mathrm{min}$ ), whereas ProW ${ }_{1-182}$-FLAG-ssrA(DD) was not $\left(\mathrm{t}_{1 / 2}>60 \mathrm{~min}\right)$. The stability of ProW $_{1-182}$-FLAG-ssrA-(DD) allowed us to show that it purified with cellular membranes but was undetectable in the soluble cytosolic fraction in fractionation experiments (Fig. 1C). As expected from results using a very similar construct, ${ }^{14}$ the activity of PhoA fusions to ProW $_{1-182}$-FLAG-ssrA confirmed that its C-terminus is cytoplasmic (Fig. 1D).

tmRNA-mediated addition of the ssrA tag in vivo can be induced using a non-stop mRNA that causes ribosome stalling at the $3^{\prime}$ end. ${ }^{3}$ When ProW ${ }_{1-182}$-FLAG was expressed from a gene with a strong transcriptional terminator and no stop codon (Fig. 2A), we observed $\sim 80 \%$ ssrA-tagged ProW ${ }_{1-182}$-FLAG (higher molecular-weight band) and $20 \%$ untagged ProW $_{1-182}$-FLAG (lower molecular-weight band) at time zero in a pulse-chase experiment (Fig. 2B; left panel). Importantly, the tagged species was degraded rapidly $\left(\mathrm{t}_{1 / 2} \sim 3 \mathrm{~min}\right.$ ) compared to the untagged species $\left(\mathrm{t}_{1 / 2} \sim 60 \mathrm{~min}\right)$ (Table 1$)$. As expected, the tagged species was not observed in a pulse-chase experiment performed in $\triangle s s r A$ cells lacking tmRNA

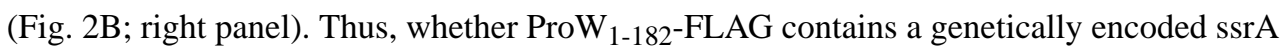
tag or acquires an ssrA tag as a consequence of ribosome stalling and tmRNA rescue, the tagged protein is rapidly degraded in E. coli.

To test the importance of different $E$. coli AAA+ proteases in intracellular degradation of ProW $_{1-182}$-FLAG-ssrA, we performed pulse-chase experiments (Fig. 3A) in cells lacking ClpP (the pep-tidase component of ClpXP and ClpAP), ClpX (the AAA+ un-foldase of 
ClpXP), ClpA (the AAA+ unfoldase of ClpAP), or FtsH (fused AAA+ unfoldase and peptidase). ProW $_{1-182}$-FLAG-ssrA was degraded at similar rates in a wild-type strain and otherwise isogenic strains lacking ClpP, ClpX, or ClpA. Thus, ClpXP and ClpAP do not contribute to intracellular degradation of ProW $_{1-182}$-FLAG-ssrA. Strikingly, however, deletion of FtsH resulted in much slower degradation of ProW ${ }_{1-182}$-FLAG-ssrA ( $t_{1 / 2}>60$ $\mathrm{min}$ ) compared to an otherwise isogenic wild-type strain $\left(\mathrm{t}_{1 / 2} \sim 5 \mathrm{~min}\right)$. We also used the non-stop stalling construct to induce ssrA tagging of ProW ${ }_{1-182}$-FLAG in cells without FtsH (Fig. 3B). The ssrA-tagged species was observed in the wild-type and $\Delta F t s H:$ Kan strains but persisted only in the latter strain $\left(t_{1 / 2} \geq 60 \mathrm{~min}\right)$. Taken together, these results show that rapid degradation of the ssrA-tagged ProW $_{1-182}$ inner-membrane protein depends upon FtsH.

Insertion of proteins into the inner membrane occurs rapidly, on time scales comparable to their rates of biosynthesis. ${ }^{16}$ For ProW $1-182$-FLAG-ssrA, synthesis should take $\sim 15$ s. To test if FtsH degrades ProW ${ }_{1-182}$-FLAG-ssrA after membrane insertion, we transformed E. coli $\Delta$ ftsH::Kan with one plasmid encoding ProW $_{1-182}$-FLAG-ssrA under IPTG control and a second plasmid encoding myc-FtsH under anhydrotetracyline (ATc) control. After briefly inducing synthesis of ProW ${ }_{1-182}$-FLAG-ssrA from $P_{\text {trc }}$ with IPTG, we added phenyl-Dgalactopyranoside (pGAL) to repress further synthesis. Two min after pGAL addition, one aliquot of cells was treated with ATc to induce myc-FtsH expression and a control aliquot was mock treated. As shown in the upper panel of Fig. 3C, when FtsH synthesis was not

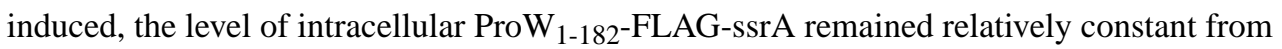
the time of pGAL addition until $\sim 40$ min. By contrast, ProW ${ }_{1-182}$-FLAG-ssrA was completely degraded after $40 \mathrm{~min}$ in cells in which myc-FtsH synthesis was induced. We

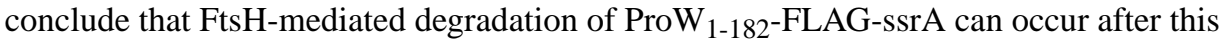
protein has been inserted into the inner membrane.

To study degradation in vitro, we purified His $_{6}$-tagged variants of FtsH and ClpXP and first verified that they efficiently degraded Arc-ssrA (Fig. 4A, right lanes), a soluble model substrate previously shown to be degraded by both proteases. ${ }^{9,17}$ Next, we tested FtsH and ClpXP degradation of purified HA-ProW ${ }_{1-182}-\mathrm{His}_{6}$-ssrA. We used this substrate because it was easier to purify than ProW $1-182$-FLAG-ssrA. FtsH and ClpXP both degraded HAProW $_{1-182}$-His $_{6}$-ssrA, but roughly $10 \mathrm{X}$ more FtsH than ClpXP was required to achieve a comparable rate of degradation (Fig. 4).

Degradation of ssrA-tagged ProW $_{1-182}$ in vivo requires FtsH but it is not affected by deletion of ClpXP or ClpAP. Degradation of ssrA-tagged ProW $_{1-182}$ in vitro was observed with both FtsH and ClpXP, but ClpXP degradation was $~ 10$-fold more efficient. Several factors could be responsible for these differences. For example, the detergent-solubilized environment of the substrate in vitro might interfere with FtsH degradation, FtsH and the substrate might need to be present in the same membrane for efficient recognition and degradation, and/or an additional cellular component or components required for efficient FtsH degradation might be missing in our reconstituted assay. The fact that ClpXP degrades ssrA-tagged ProW $\mathrm{W}_{1-182}$ in vitro but not in vivo is likely to be a consequence of the substrate being embedded in the inner-membrane in vivo, which could reduce accessibility of the ssrA tag and/or create an energetic barrier that is too high for ClpX to pull ssrA-tagged ProW $_{1-182}$ out of the 
membrane. Further studies will be required to distinguish between these mechanistic possibilities.

\section{Supplementary Material}

Refer to Web version on PubMed Central for supplementary material.

\section{Acknowledgments}

Funding Sources

This work was supported by NIH grant R01 AI-016892 (R.T.S.) and by a Ruth L. Kirschstein National Research Service Award (F32GM116241) (S.B.H.).

We are grateful for E. coli strains provided by Teru Ogura. We thank Karl Schmitz, Juhee Park, and Ohad Iosefson for helpful suggestions.

\section{References}

1. Keiler KC. Mechanisms of ribosome rescue in bacteria. Nat Rev Microbiol. 2015; 13:285-297. [PubMed: 25874843]

2. Gottesman S, Roche E, Zhou Y, Sauer RT. The ClpXP and ClpAP proteases degrade proteins with carboxy-terminal peptide tails added by the SsrA-tagging system. Genes Dev. 1998; 12:1338-1347. [PubMed: 9573050]

3. Keiler KC, Waller PR, Sauer RT. Role of a peptide tagging system in degradation of proteins synthesized from damaged messenger RNA. Science. 1996; 271:990-993. [PubMed: 8584937]

4. Sin C, Chiarugi D, Valleriani A. Quantitative assessment of ribosome drop-off in E. coli. Nucleic Acids Res. 2016; 44:2528-2537. [PubMed: 26935582]

5. Sauer RT, Baker TA. AAA+ proteases: ATP-fueled machines of protein destruction. Annu Rev Biochem. 2011; 80:587-612. [PubMed: 21469952]

6. Farrell CM, Grossman AD, Sauer RT. Cytoplasmic degradation of ssrA-tagged proteins. Mol Microbiol. 2005; 57:1750-1761. [PubMed: 16135238]

7. Lies M, Maurizi MR. Turnover of endogenous SsrA-tagged proteins mediated by ATP-dependent proteases in Escherichia coli. J Biol Chem. 2008; 283:22918-22929. [PubMed: 18550539]

8. Akiyama Y, Kihara A, Ito K. Subunit a of proton ATPase F0 sector is a substrate of the FtsH protease in Escherichia coli. FEBS Lett. 1996; 399:26-28. [PubMed: 8980112]

9. Herman C, Prakash S, Lu CZ, Matouschek A, Gross CA. Lack of a robust unfoldase activity confers a unique level of substrate specificity to the universal AAA protease FtsH. Mol Cell. 2003; 11:659669. [PubMed: 12667449]

10. Kihara A, Akiyama Y, Ito K. FtsH is required for proteolytic elimination of uncomplexed forms of SecY, an essential protein translocase subunit. Proc Natl Acad Sci U S A. 1995; 92:4532-4536. [PubMed: 7753838]

11. Kihara A, Akiyama Y, Ito K. Different pathways for protein degradation by the FtsH/HflKC membrane-embedded protease complex: an implication from the interference by a mutant form of a new substrate protein, YccA. J Mol Biol. 1998; 279:175-188. [PubMed: 9636708]

12. Herman C, Thevenet D, Bouloc P, Walker GC, D'Ari R. Degradation of carboxy-terminal-tagged cytoplasmic proteins by the Escherichia coli protease HflB (FtsH). Genes Dev. 1998; 12:1348_ 1355. [PubMed: 9573051]

13. Chai Q, Wang Z, Webb SR, Dutch RE, Wei Y. The ssrA-Tag Facilitated Degradation of an Integral Membrane Protein. Biochemistry. 2016; 55:2301-2304. [PubMed: 27078234]

14. Haardt M, Bremer E. Use of phoA and lacZ fusions to study the membrane topology of ProW, a component of the osmoregulated ProU transport system of Escherichia coli. J Bacteriol. 1996; 178:5370-5381. [PubMed: 8808924] 
15. Whitley P, Zander T, Ehrmann M, Haardt M, Bremer E, von Heijne G. Sec-independent translocation of a 100-residue periplasmic N-terminal tail in the E. coli inner membrane protein proW. EMBO J. 1994; 13:4653-4661. [PubMed: 7925306]

16. Ito K, Sato T, Yura T. Synthesis and assembly of the membrane proteins in E. coli. Cell. 1977; 11:551-559. [PubMed: 328166]

17. Burton RE, Siddiqui SM, Kim YI, Baker TA, Sauer RT. Effects of protein stability and structure on substrate processing by the ClpXP unfolding and degradation machine. EMBO J. 2001; 20:30923100. [PubMed: 11406586] 

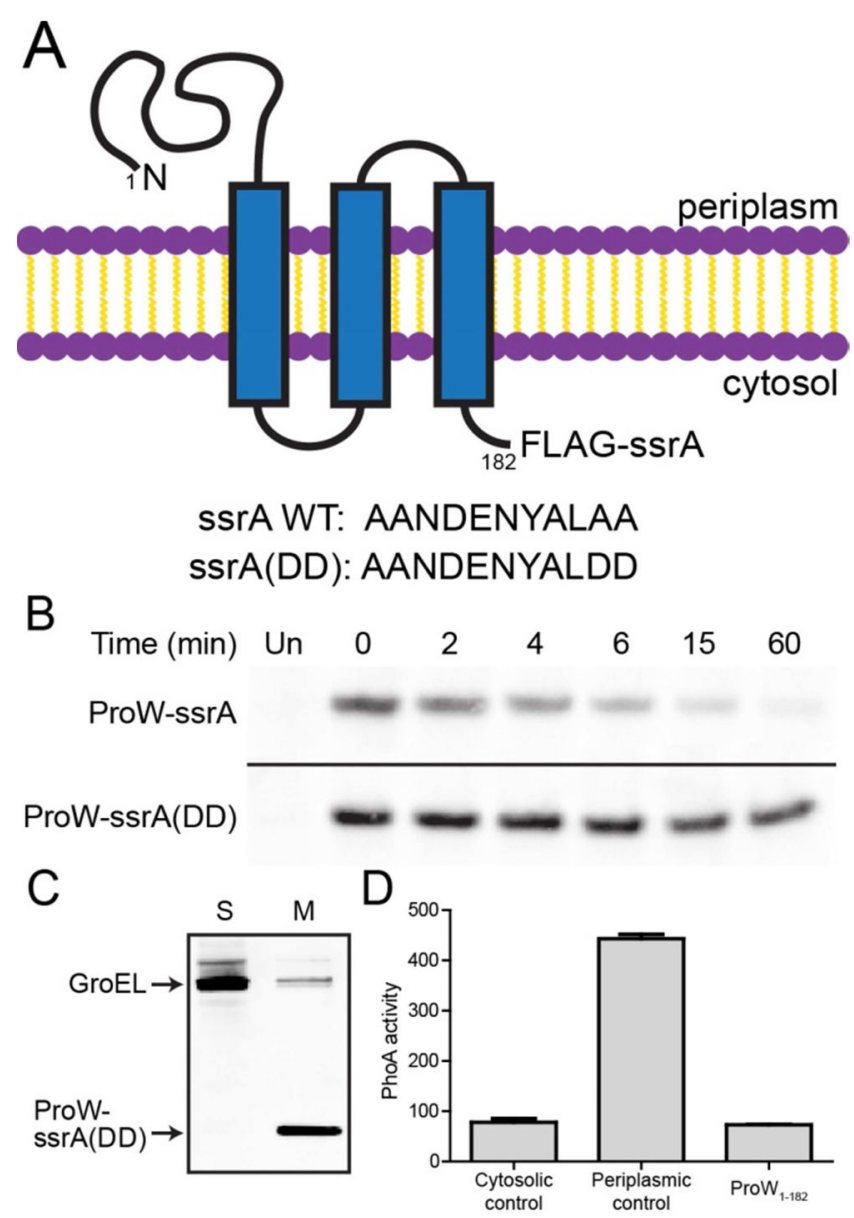

Figure 1.

An ssrA-tagged model membrane protein is degraded in E. coli. (A) Topology of ProW 1-182- $^{-}$ FLAG-ssrA (top) and amino-acid sequences of wild-type and mutant ssrA tags (bottom). (B) Expression of ProW 1-182-FLAG-ssrA or ProW 1 182-FLAG-ssrA(DD) in E. coli X90 was induced, pulsed with ${ }^{35}$ S-labeled methionine and cysteine, and chased with unlabeled amino acids. Samples taken at different times were immunoprecipitated and analyzed by SDSPAGE and autoradiography. "Un" indicates an uninduced control sample. (C) E. coli cells expressing ProW 1 -182-FLAG-ssrA(DD) were lysed and fractionated into soluble $(\mathrm{S})$ and membrane $(\mathrm{M})$ fractions, which were analyzed by SDS-PAGE and immunoblotted using antibodies against GroEL (a cytosolic protein) and the FLAG tag. (D) Activity (in Miller units) of C-terminal FLAG-ssrA-PhoA fusions of a cytosolic protein ( $\lambda$ repressor), a periplasmic protein (cytochrome $\mathrm{b}_{562}$ ), and ProW $1-182$. 


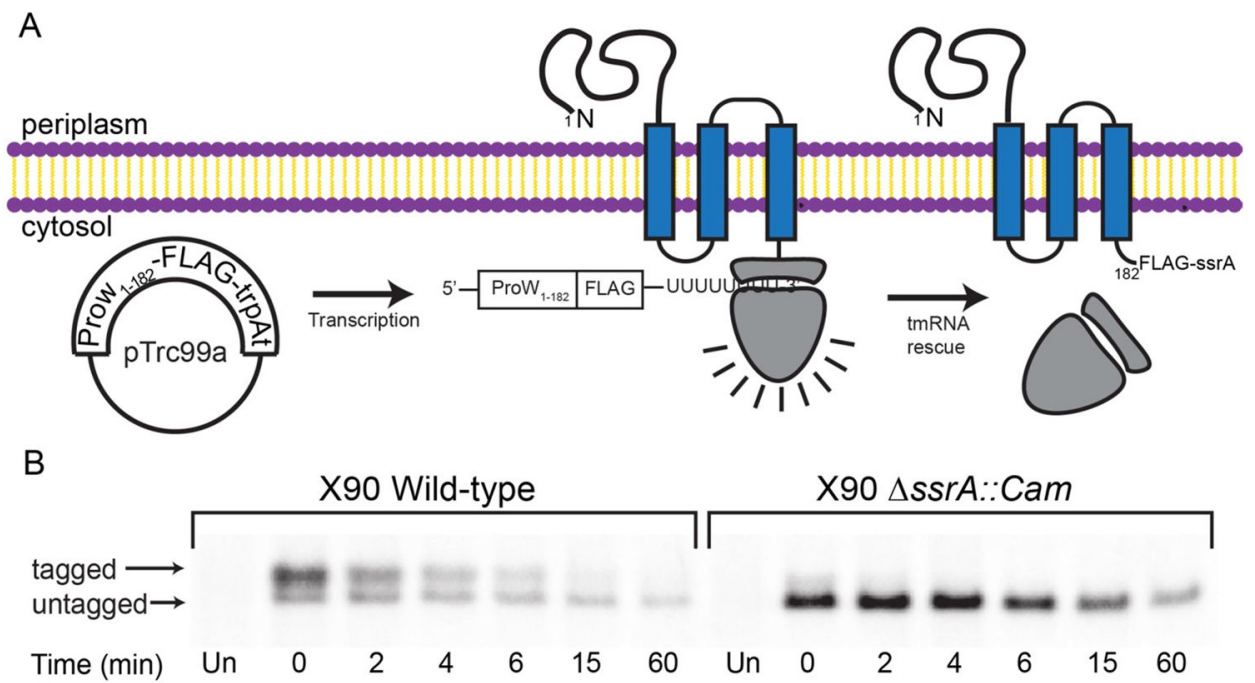

Figure 2.

tmRNA-mediated tagging of ProW $\mathrm{W}_{1-182}$ results in rapid degradation. (A) A non-stop mRNA encoding ProW $\mathrm{W}_{1-182}$-FLAG results in ribosomal stalling and ssrA tagging. (B) A plasmid encoding ProW 1 -182-FLAG-trpAt was transformed into wild-type E. coli X90 or an otherwise isogenic $\triangle \operatorname{ssr} A:$ Cam strain, and the half-life of the tagged and untagged proteins was measured by pulse-chase experiments as described in Fig. $1 \mathrm{~B}$. 


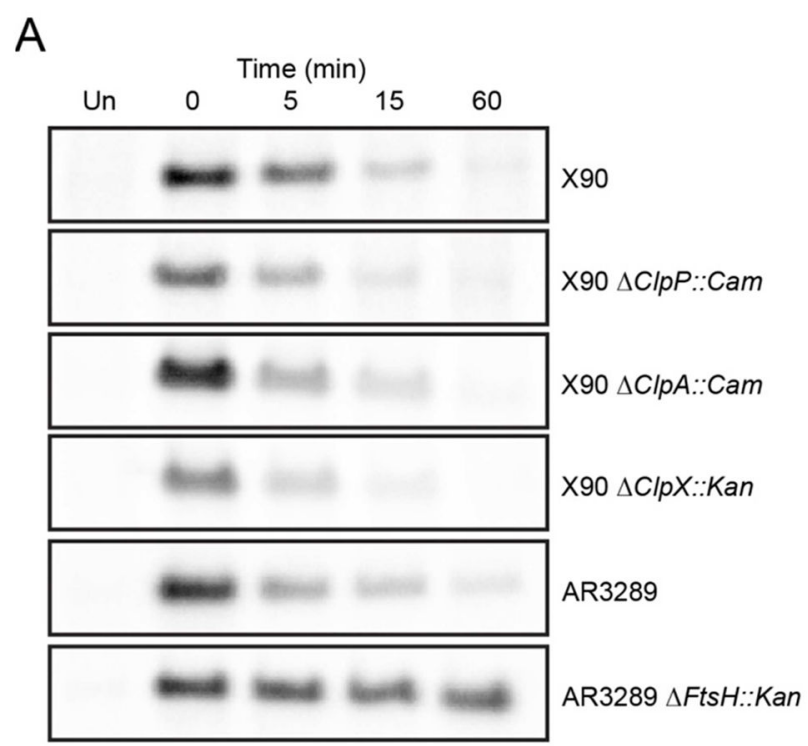

B

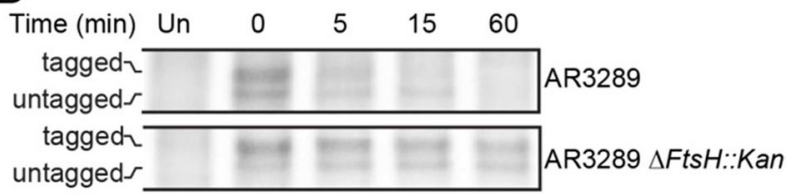

C

ProW $_{1-182}$-FLAG-ssrA induction
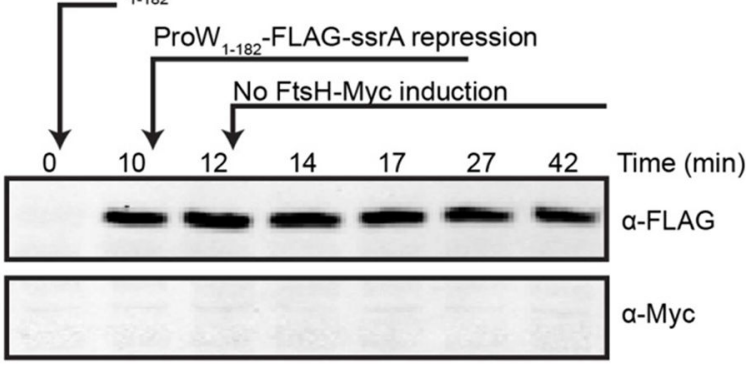

+ FtsH-Myc induction

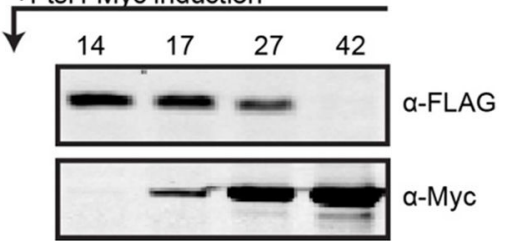

Figure 3.

FtsH degrades ProW 1-182-FLAG-ssrA. (A) Pulse-chase experiments demonstrate that ProW 1 -182-FLAG-ssrA has a longer half-life in cells lacking FtsH compared to wild-type cells or those lacking ClpP, ClpX, or ClpA (see Fig. 1B legend for experimental details). (B) Auto-radiograms of whole-cell lysates from pulse-chase experiments show that endogenously ssrA-tagged ProW $1-182$-FLAG-trpAt is also stabilized in a strain lacking FtsH. (C) Transient induction of plasmid-encoded ProW 1-182-FLAG-ssrA in E. coli AR3289 $\Delta f t s H:$ Kan resulted in little degradation (top panels) unless expression of plasmid-encoded Myc-tagged FtsH was subsequently induced (bottom panels). In all panels, ProW $1-182^{-}$ 
FLAG-ssrA expression was induced with IPTG for $10 \mathrm{~min}$, and then pGAL was added to repress further expression. In the bottom panels, expression of Myc-tagged FtsH was induced by addition of ATc two min after addition of pGAL. Samples were separated by SDS-PAGE and immunoblotted with anti-FLAG and anti-Myc tag antibodies. 

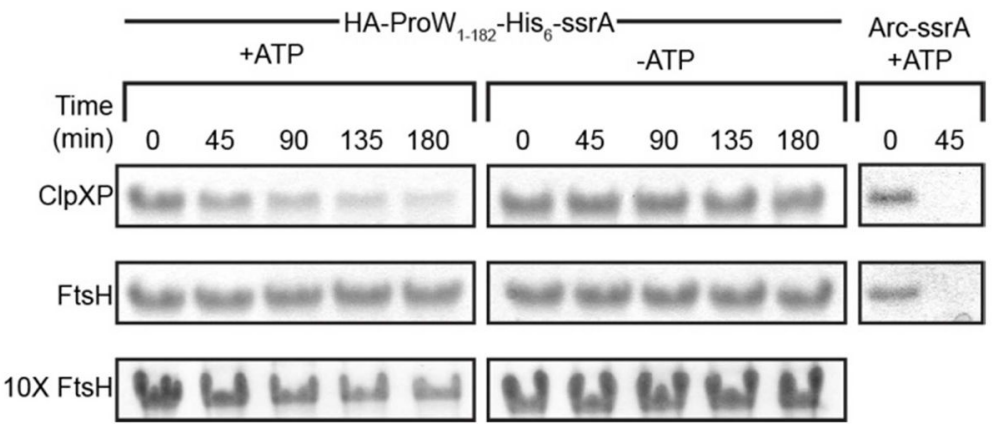

Figure 4.

In vitro degradation of a model membrane protein. Purified HA-ProW $1-182-\mathrm{His}_{6}$-ssrA was degraded by purified ClpXP (top panel) or FtsH (middle and bottom panels) in ATP-

dependent reactions. Arc-ssrA was also degraded by both proteases. Reactions were initiated by addition of $4 \mathrm{mM}$ ATP (with regeneration system), quenched at different times, and then analyzed by SDS-PAGE and staining with Coomassie Blue. 


\section{Table 1}

Half-lives of ProW constructs determined from two independent experiments. n.d. - not determined.

\begin{tabular}{|c|c|c|}
\hline & \multicolumn{2}{|c|}{ Half-life (min) } \\
\hline & $\mathbf{X 9 0}$ & X90 $\Delta s s r A::$ Cam \\
\hline ProW $_{1-182}$-FLAG-ssrA & 7,4 & n.d. \\
\hline ProW $_{1-182}$-FLAG-ssrA(DD) & $>60,>60$ & n.d. \\
\hline ProW $_{1-182}$-FLAG-trpAt & 3,2 & $\sim 60, \sim 60$ \\
\hline
\end{tabular}

